\title{
VULNERABILIDAD SOCIAL DE LA NIÑEZ MIGRANTE NO ACOMPAÑADA: UNA MIRADA DESDE EL ÁREA URBANA DE ALTAR, SONORA, MÉXICO
}

\section{SOCIAL VULNERABILITY OF UNACCOMPANIED MIGRANT CHILDREN: A VIEW FROM THE URBAN AREA OF ALTAR, SONORA, MEXICO}

Mario Alexander Cabrera Duarte* y Gloria Ciria Valdéz Gardea**

Resumen: Este artículo es producto de la investigación desarrollada por los autores, sobre la vulnerabilidad social de la niñez migrante no acompañada, en el área urbana del municipio de Altar, Sonora, durante los años 2010-2011. Las técnicas principales utilizadas para la recolección de los datos fueron la observación participante y la entrevista semiestructurada.

Los resultados of recidos se limitan a evidenciar la vulnerabilidad social que sufre la niñez migrante no acompañada, al hacer uso de los servicios de alimentación, hospedaje y salud. Lo cual les expone a una serie de riesgos, como ser la escasez de alimento, la perdida de sus pocas pertenencias, al consumo de drogas, a las agresiones físicas, al padecimiento de enfermedades y a un limitado acceso a la atención médica.

\section{Maestro en Ciencias Sociales.}

Docente del Centro Universitario de Educación a Distancia de la Universidad Pedagógica Nacional Francisco Morazán de Honduras. Actualmente cursa el Doctorado en Filosofía que ofrece la Universidad de Valladolid, España.

ratioupn@yahoo.es

** Doctora en Antropología Cultural.

Docente investigadora y coordinadora del Seminario Niñez Migrante en El Colegio de Sonora, México.

gvaldez@colson.edu.mx 
Palabras clave: niñez migrante no acompañada, vulnerabilidad social, alimentación, hospedaje, salud.

Abstract: This article is the result of research carried out by the authors on the social vulnerability of unaccompanied migrant children in the urban area of Altar, Sonora, during the years 20102011. The main techniques used for data collection were participant observation and semi-structured interview.

The results offered are limited to evidence the social vulnerability suffered by unaccompanied migrant children, by making use of the services of food, accommodation and health. Which exposes them to a number of risks, such as food shortages, the loss of their few belongings, the drug, the physical, the suffering of diseases and limited access to medical care aggressions.

Keywords: unaccompanied migrant children, social vulnerability, feeding, lodging, health.

\section{INTRODUCCIÓN}

En los últimos años los estudios sobre migración internacional, se han enfocado en visibilizar la presencia de un sector poblacional antes no documentado, como lo es el de la niñez migrante. La que se ha hecho visible al migrar junto a sus familias o de manera no acompañada, integrándose también a lo que se denomina como migración de retorno (López Castro, 2005; Gallo Campos, 2004; Rangel Gómez, 2008; Valdéz Gardea, 2007, 2008, 2009; Chavez \& Menjívar, 2010).

La niñez se ha involucrado en la migración indocumentada de distintas maneras, existen niños y niñas que se quedan en la comunidad mientras uno o ambos padres migran, otros deciden emprender el viaje acompañados o solos por diversos motivos (reunificación familiar, trabajo, curiosidad etc.); también están los que nacieron en Estados Unidos de América y son hijos de migrantes, además de ellos, recientemente han surgido con mayor fuerza, los que retornan a su país de forma voluntaria o no.

La niñez migrante representa un desafío para la administración pública mexicana, las entidades vinculadas al tema han creado varias iniciativas para protegerles, entre las que se encuentran el programa de repatriación liderado por los albergues Camino a Casa que 
forman parte de los Sistemas Estatales para el Desarrollo Integral de la Familia (SEDIF), y los Oficiales de Protección a la Infancia pertenecientes al Instituto Nacional de Migración (INM), donde confluyen los esfuerzos de otras instituciones gubernamentales y organizaciones de la sociedad civil.

Sin embargo, las iniciativas implementadas por el gobierno y las organizaciones que les apoyan, se encuentran limitadas por la escasa existencia de información sistematizada que permita una mayor comprensión del fenómeno, y la intervención integral que asegure la efectividad de sus acciones.

Hasta el 2010 la mayoría de las investigaciones realizadas, tendían a centrarse en las experiencias de los adultos (Chavez \& Menjivar, 2010: 75) generándole a la niñez una imagen de actores pasivos, con limitada capacidad en la construcción de su realidad migratoria; estereotipo que se debe romper ya que ellos evidencian con sus propias voces o mediante otras fuentes de información, lo vivido en el proceso migratorio.

En este artículo se brinda un acercamiento a la vulnerabilidad social de la niñez migrante no acompañada de origen mexicano, a partir de la experiencia que vivieron los niños y niñas que fueron entrevistados, así como observados, al hacer uso de los servicios de alimentación, hospedaje y salud que se prestan en el área urbana de Altar, Sonora, cuando intentaban ingresar sin documentos a Estados Unidos de América.

\section{APROXIMACIÓN TEÓRICA}

Las categorías de análisis principales en torno a las que giró la investigación fueron la de niñez migrante no acompañada, y la de vulnerabilidad social. El término niñez migrante no acompañada se utiliza para designar a «todo ser humano menor de 18 años de edad» (Fondo de las Naciones Unidas para la Infancia, 2006: 10) «que se encuentra [separado] de ambos padres u otros parientes, y que en el proceso migratorio no está bajo el cuidado de ningún adulto, que por ley a costumbre, [lo tenga] a su cargo» (Alto Comisionado de las Naciones Unidas para los Refugiados, 2009: 2).

La utilización de esta categoría de análisis es eminentemente técnica, ya que durante el recorrido, la niñez migrante siempre se 
hace acompañar de alguien, que puede ser otro niño o niña que también migra, un coyote $^{1}$ o un amigo de la familia, los que en ningún momento son responsables legales de su tutela.

La vulnerabilidad social es la segunda categoría principal de análisis, la cual se ha convertido en «un tema pendiente por resolver [en] la problemática de los flujos migratorios a nivel mundial» (Santos Villareal \& Ávila Loya, 2009: 11). En el caso de la niñez migrante, esta es un "rasgo social intrínseco» (González Fagoaga, 2009: 233) caracterizado de acuerdo a Bustamante (2004: 309) por la ausencia de empoderamiento para enfrentar y protegerse de los riesgos a los que se expone.

Pizarro (2001: 11-12) considera que en la definición del término vulnerabilidad social, han tenido gran influencia las siguientes vertientes académicas que son especialmente de origen anglosajón: la que se ha dedicado a estudiar los desastres naturales, haciendo énfasis en aspectos físicos; y la que trata de comprender los cambios en las condiciones de vida que experimentan las comunidades rurales pobres, ante los eventos socioeconómicos traumaticos.

Tener como elementos centrales de la vulnerabilidad social los desastres naturales y los eventos socioeconomicos traumaticos, la vuelve poco funcional para estudiar con una perspectiva cualitativa, temas vinculados con migración, salud, educación, o aspectos juridicos; en los cuales las personas están más propensas a ser dañadas por otros, en función al rol social que desempeñan.

$\mathrm{Si}$ bien es cierto que los aportes de las vertientes anteriores han sido significativos, los trabajos desarrollados en América Latina «aunque con distintos grados de sistematización»(Busso, 2001: 8) nos plantean el surgimiento de una tercera vertiente. En ella no se deja de lado los aspectos físicos-económicos, pero está centrada, en aquello que puede causar daño, o contribuir a que esto suceda a las personas y determinadas comunidades, por el rol social que desempeñan, la estigmatización a la que se ven sometidas, así como por el establecimiento de relaciones sociales asimétricas.

Desde la vertiente latinoamericana, la vulnerabilidad social no solo es la indefensión ante los eventos que pueden causar daño, en

1 En la jerga utilizada por los diferentes actores sociales vinculados con la migración indocumentada de la población mexicana hacia Estados Unidos de América, se le denomina «Coyote» $\mathrm{o}$ «Pollero», a quien se encarga de coordinar el traslado del migrante desde su lugar de origen hasta donde pretende llegar, cuando lo hace dentro de una organización —ilegal— que se dedica a esta actividad. 
ella estan implicitas «un conjunto de situaciones [...] que afectan la convivencia» (Almeida Acosta, 2009: 65) entre individuos, creando una «generalizada percepción de incertidumbre, inseguridad, desprotección, precariedad o perdida de derechos» (Comisión Económica para América Latina y el Caribe, 2001: 6), que aumenta al presentarse problemas económicos, o desastres naturales. Esto hace que las personas se vean limitadas "para aprovechar las oportunidades disponibles, [...] mejorar su situación de bienestar [e] impedir su deterioro» (Kaztman, 2000: 281), en el menor tiempo posible.

En el proyecto de investigación que dio origen a este artículo, la vulnerabilidad social se entendió como la inseguridad, precariedad, e indefesión a la que está sometida la niñez migrante no acompañada, producto de las relaciones sociales asimetricas que establece con los vecinos de los lugares de paso en los que pernocta temporalmente, las que se mantienen y fortalecen debido a la política antiinmigrante implementada en la frontera por el país receptor, y por la incapacidad institucional del país expulsor para prevenir la integración de su niñez a este fenómeno social.

Desde esta perspectiva, surge "como una configuración negativa entre aspectos micro y macro sociales» (Comisión Económica para América Latina y el Caribe, 2001: 8) que afectan a la persona, los que se manifiestan a partir de las acciones sociales establecidas entre quienes la sufren, y los que contribuyen a provocarla mediante su participación activa o pasiva.

Entendida así, la vulnerabilidad social está ligada al riesgo, el cual según Beck (1998: 34) supone la pérdida de la seguridad, y la probabilidad de que la persona sufra algún daño en el contexto que se encuentra. Este riesgo es producto de la construcción social y cultural, ${ }^{2}$ la que se vincula con aspectos subjetivos del tejido social, que influyen para que se reduzca o aumente, de acuerdo a las acciones de las personas, grupos sociales o el Estado ${ }^{3}$.

2 Para ahondar más sobre la construcción social y cultural del riesgo véase a: Ruiz, O. (1998). Riesgo, migración y espacio fronterizo: Una reflexión. Pág. 261

3 Para conocer otros enfoques de lo que es la vulnerabilidad social véase a: Busso, G. (2001). Vulnerabilidad social: Naciones e implicaciones de politicas para Latinoamérica a inicios del siglo XXI. Págs. 37-38. 


\section{METODOLOGÍA DE LA INVESTIGACIÓN}

La investigación que dio origen a este documento, tenía por objetivo describir los aspectos existentes en los servicios de alimentación, hospedaje y salud prestados en el área urbana de Altar, Sonora que podían vulnerar a la niñez migrante no acompañada.

La metodología empleada durante todo el proceso de investigación fue la cualitativa, debido a que cuenta con las técnicas adecuadas que permiten comprender bajo qué condiciones se relaciona la niñez migrante no acompañada con los habitantes de los lugares que transitan. La metodología cualitativa trasciende la idea de ver a los niños y niñas como simples datos estadísticos, para tratar de captar las dinámicas relaciones micro-sociales que generan en el entorno que se encuentran.

Su uso es importante para el estudio de migrantes en movimiento porque brinda la oportunidad de «comprender el sentido de las acciones humanas» (Denman \& Haro, 2000: 39) a través de los datos descriptivos que genera, los que se obtienen en especial al tomar en cuenta las palabras pronunciadas o escritas de la gente y lo observado (Taylor \& Bogdan, 1992: 152) en los escenarios donde se dan los hechos sociales estudiados.

Otras características que tiene, es que ayuda a «comprender los valores culturales y representaciones de determinados grupos sobre temas específicos» (De Sauza Minayo, 1995: 115), sensibiliza al investigador ante "los comportamientos, las actitudes y las formas de vida» (Velasco \& Díaz de la Rada, 2006: 29) de los sujetos vinculados en el tema de estudio, además contribuye a visibilizar los problemas sociales "para reparar desigualdades y apoyar de [manera] específica, a poblaciones marginadas intencional o accidentalmente» (Figueroa, 2003: 67) como sucede en el caso de la migración indocumentada.

Las actividades desarrolladas se pueden agrupar en lo que la antropología a considerado tradicionalmente como el trabajo de gabinete y el de campo; el trabajo de gabinete incluyó la revisión de documentos académicos, notas periodísticas, informes y reportes técnicos, de instituciones públicas locales, nacionales y organismos internacionales que brindaron un acercamiento al fenómeno y la localidad estudiada; en el mismo se organizó, y analizó la información recolectada que dio origen a los resultados obtenidos.

El trabajo de campo, consistió en la implementación de la 
observación participante durante 31 días, y la obtención de 37 entrevistas semiestructuradas, de las cuales 16 fueron aplicadas a personas adultas que se encontraban durante ese tiempo en la localidad, entre ellas se incluyen migrantes, líderes religiosos, pequeñas comerciantes, funcionarios públicos municipales y estatales. Las 21 entrevistas restantes se aplicaron a niños y niñas migrantes, que pernoctaron temporalmente en el área urbana de Altar durante su recorrido migratorio.

La observación participante y la entrevista semiestructurada son unas de las técnicas más usadas en forma conjunta (De Sauza Minayo, 1995; Denzin, 2002) al realizar investigaciones cualitativas con enfoque etnográfico, debido a que permiten obtener «el punto de vista [...] y la historia del entrevistado»(Galindo, 1987: 156), visibilizando a los sujetos estudiados, como actores de los hechos sociales que narran mediante su propia voz.

La muestra fue seleccionada mediante la técnica de la bola de nieve ${ }^{4}$, el entrevistador acudía a los entrevistados para que sugirieran a otras personas que podían ser entrevistadas. En el caso de la niñez debía cumplir el requisito de haber pernoctado por lo menos una noche en el área urbana de Altar, tener entre 14 y 17 años de edad, y estar migrando sin documentos. Mientras que los adultos seleccionados estaban vinculados a las instituciones públicas, o religiosas encargadas de brindarles algún tipo de atención.

Otros aspectos que influyeron para determinar el tamaño de la muestra, fue el tiempo que duró la observación participante, la disponibilidad de las personas a quienes se les solicitaba participar, y la saturación generada por los datos obtenidos.

El enfocar la investigación en la niñez migrante con una edad comprendida entre los 14 y 17 años, se debió a que es el segmento de este grupo poblacional que más migra, en el 2010 de acuerdo al Instituto Nacional de Migración (2010: 152) participaron en el 91 por ciento de los eventos de repatriación.

$4 \quad$ Al trabajar con población que se encuentra en su proceso migratoria la técnica de la bola de nieve es sumamente útil, ya que esta como lo expresan Martín-Crespo Blanco \& Salamanca Castro (2007: 2) permite pedirle a los informantes que recomienden a otros posibles participantes, además reduce los costos económicos, facilita el acceso a personas difíciles de identificar, y contribuye a establecer con mayor facilidad una relación de confianza entre el investigador y los investigados. 


\section{INCORPORACIÓN DE LA NIÑEZ MEXICANA A LA MIGRACIÓN INDOCUMENTADA HACIA ESTADOS UNIDOS DE AMÉRICA}

La migración indocumentada de mexicanos a Estados Unidos de América, muestra la incorporación de diferentes sectores poblacionales a este fenómeno social. Antes de que se implementara en el país receptor la Ley de Reforma y Control de la Inmigración aprobada en 1986 (Tuirán \& Luis, 2010), los migrantes eran en su mayoría hombres, pero en esta segunda década del siglo XXI, los datos estadísticos evidencian la presencia de miles de mujeres y menores de edad que anualmente intentan ingresar sin documentos al territorio estadounidense; entre los años 1998 y 2010 el Estado mexicano realizó 680.550 eventos de repatriación (Instituto Nacional de Migración, 2011: 11) de niños y niñas migrantes.

\section{GRÁFICO 1}

EVENTOS DE REPATRIACIÓN DE NIÑOS Y NIÑAS MIGRANTES DESDE ESTADOS UNIDOS DE AMÉRICA EN EL PERIODO COMPRENDIDO ENTRE 1998 Y EL 2010

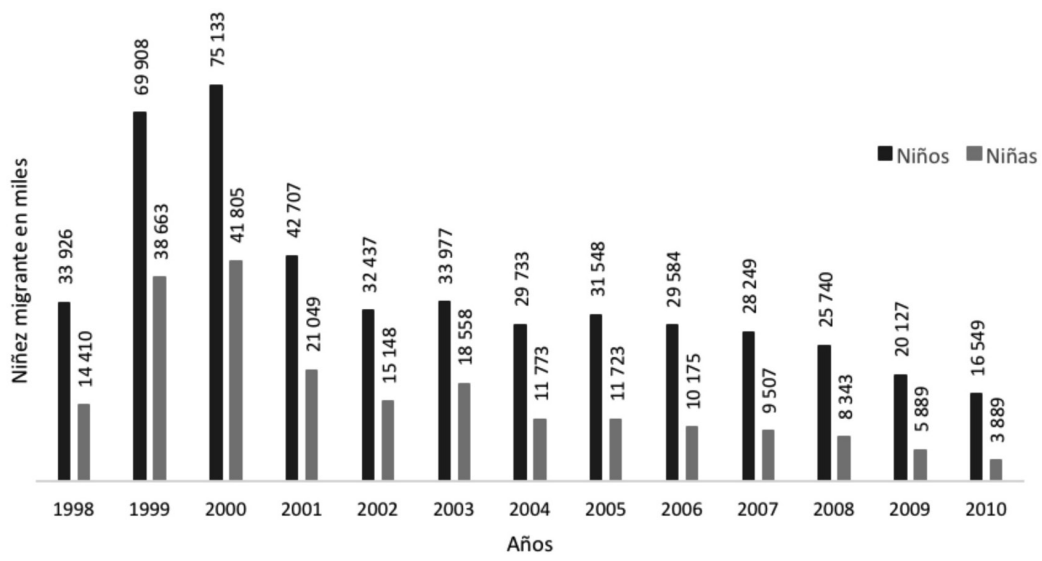

Fuente: elaboración propia en base a las series históricas del Instituto Nacional de Migración (2011: 1). De 1998 al 2010 el Estado mexicano repatrió 469.618 niños y 210.,932 niñas, estos datos son una de las pocas evidencias oficiales a las que se puede acceder, sobre la participación de la niñez mexicana en la migración indocumentada internacional. 
La niñez mexicana tiene menos de dos décadas de haberse incorporado como sujetos activos, y en grandes cantidades a la migración indocumentada rumbo a Estados Unidos de América, «en especial los de 14 a 17 años de edad» (Valdéz Gardea 2008: 16). Lo que se ha facilitado gracias a las bondades generadas por uno de los pilares fundamentales de la globalización, como lo es la Tecnología de la Información y la Comunicación (TIC).

En ellos la decisión de migrar es causada por una variedad de factores, entre los que se encuentran la presión de otros familiares que ya han viajado de la misma forma, la demanda de trabajadores de la sociedad receptora, la penetración capitalista en sus comunidades, las redes migratorias y el surgimiento de organizaciones humanitarias (Massey et al., 2008; Sufcliffe, 1998) que les protegen, lo que se vincula a las «condiciones estructurales de pobreza, exclusión social y violencia» (García Vidales, Molina Nava, \& López Vega, 2008: 52-53) que viven.

A esto también se suma la reunificación familiar, el deseo de experimentar o comprobar si es cierto lo que se dice de la migración indocumentada, y hasta el utilizarla para los ritos de paso como sucede en San Andrés Huixtac, Guerrero; aquí la niñez migra para buscar a sus padres y hermanos, o pasar a otra etapa dentro de su sociedad, lo que también se da en San Juan Caltimacán, Hidalgo, en donde expresan que cuando un muchacho prueba el norte, se vuelve hombre (Yánez Moreno \& Hernández Martínez, 2010: 7-8).

La presencia de la niñez en la migración indocumentada hacia Estados Unidos de América, es una realidad insoslayable, y aunque los datos generados por el Instituto Nacional de Migración (2011: 1) como resultados de los eventos de repatriación, muestran una tendencia a la baja del $83 \%$ entre el año 2000 y el 2010, no se puede garantizar con exactitud que la cantidad de los que migran sin documentos, se está reduciendo aceleradamente. Debido a que el mayor control fronterizo, ha generado una transformación en la manera de operar de las organizaciones que se encargan de traficar con los migrantes indocumentados. Además, algunos de los que migran mueren en el desierto por las duras condiciones climáticas, y otros de acuerdo a Amnistía Internacional (2010: 11-20) son secuestrados o asesinados durante su recorrido. 


\section{EL ÁREA URBANA DE ALTAR COMO LUGAR DE PASO MIGRATORIO}

Altar desde que se fundó «ha sido tierra de paso, cruce de caminos, y puerta de entrada al inmenso desierto que cubre parte de lo que hoy es el norte de Sonora y el sur de Arizona» (Von der Borch, 2011: 3). En el siglo XIX ya se empezaba a documentar la presencia de migrantes que pernoctaban por un tiempo, y luego continuaban su recorrido hacia otros pueblos, en 1873 sus autoridades mostraban preocupación por lo que estaba sucediendo; y mediante un informe con fecha del 26 de febrero de ese año, el prefecto le comunicaba al secretario de Estado de Ures lo siguiente: «Pongo de su conocimiento de esa secretaría, que en los últimos días han llegado á esta villa ciento y tantas personas de ambos sexos, con dirección unos a la Alta California, y otros se han dividido (sic) en las diversas municipalidades del distrito» (Lizárraga García, 2000: 371).

En la segunda década del siglo XXI, la localidad continúa siendo utilizada por los migrantes, solo que ahora en su mayoría, son personas que intentan ingresar sin documentos a Estados Unidos de América por la ruta Altar-El Sásabe. De acuerdo a Lizárraga García (2000: 371) ellos comenzaron hacerse visibles a partir de 1980, cuando llegaban del interior de la república, se les miraba uno o dos días y luego desaparecían.

A partir de 1994 su presencia aumentó sin precedente, para ese año se estimaba que a diario llegaban 300 personas, en el 2000 alcanzaron las 1.200 (Francisco, entrevista, 2006) y en el 2011 se redujeron a 250; la baja presentada fue influenciada por la crisis económica, las políticas antiinmigrantes en Arizona, y los abusos cometidos por la mafia en esta área de la línea fronteriza.

La fluctuación del número de personas, generada por los que llegan y salen, imposibilita a las instituciones públicas, determinar con exactitud la cantidad poblacional del espacio municipal. Por ejemplo, el 2010 se anunciaba la existencia de 9.049 habitantes (Instituto Nacional de Estadística y Geografía 2011), pero Mendoza Rockwell (2006: 34) expresa en su tesis de licenciatura, que por lo menos 10 años antes del 2006, un letrero en la entrada del pueblo reportaba un total de 14.930 .

El área urbana de Altar se ha convertido en una de las principales antesalas de espera para las personas que intentan cruzar la frontera. A pesar de ser pequeña, en los últimos años se encuentra abierta 
a personas de todo el mundo (Careaga, 2009: 127), entre los que la transitan y pernotan, se encuentran mexicanos procedentes de Veracruz, Oaxaca, Chiapas, Guerrero, Puebla, Tlaxcala, Sinaloa, Sonora y Baja California; además de los migrantes internacionales procedentes de Guatemala, El Salvador, Honduras, Nicaragua, medio oriente (árabes e israelitas) y China, a estos últimos localmente se les conoce como "pollos exóticos», por los altos precios que deben pagar a las redes que trafican con indocumentados, para que los lleven al lugar que tienen previstos.

Con la migración indocumentada, este pueblo evolucionó en sus actividades económicas, pasando de la agricultura y ganadería a la prestación de servicios, hubo gente que se desarrolló, gracias al dinero dejado por los migrantes antes de irse (Valdéz Gardea, notas de campo, 2006); lo que también provocó una transformación de su fisonomía, por la aparición de los negocios de comida, ropa, provisiones (Truax, 2006) para llevar durante el recorrido, comunicación, hospedaje, y por el surgimiento de una flota de transporte terrestre que lo conecta con El Sásabe y al resto del país.

Los espacios comerciales para brindarles servicios a los que pernoctan en él, crecieron de forma exponencial, hace unos 30 años solo existían dos hoteles (el Marielena y el San Francisco), junto a negocios emblemáticos como la tienda de ropa Moraga y la papelería Mary. En el 2011 habían 10 casas de cambio y empeño, 18 hoteles, 10 casetas telefónicas, 10 cantinas y expendios, 43 «tianguis» (venta de ropa y zapatos), 50 abarroterías, 8 farmacias (Ayuntamiento de Altar 2010); y se consideraba que existían más de un centenar de casas de huéspedes, a parte de las de seguridad, de estas últimas se conoce muy poco, las personas evitan hablar de ellas por el temor que les genera.

El auge migratorio provocó que las instituciones vinculadas con servicios prioritarios como salud y seguridad tuvieran nuevos usuarios, además aparecieron dos sectores influyentes que desarrollan sus actividades en torno al migrante indocumentado, uno es la mafia y el otro el voluntariado que lucha por la defensa de sus derechos humanos, dentro del cual sobresale el Centro Comunitario de Atención al Migrante y Necesitado (CCAMYN) de la iglesia católica, por su nivel de organización y la cantidad de personas que aglutina.

Aunque existen iniciativas públicas como la oficina municipal para el Desarrollo Integral de la Familia (conocida popularmente 
con el nombre de DIF Municipal) ${ }^{5}$, la Dirección General de Atención a Migrantes Internacionales (DGAMI) y la Comisión Nacional de Derechos Humanos (CNDH), que realizan actividades en favor del migrante, la institución que mantiene un programa permanente para atenderles de manera inmediata es la iglesia católica Nuestra Señora de Guadalupe, con el CCAMYN.

Quienes lo utilizan, encuentran no solo un espacio en donde dormir; aparte de sus habitaciones con capacidad para 40 personas, tienen acceso al desayuno y la cena, además de medicina, duchas, sanitarios, ropa, orientación sobre los peligros que corren en el desierto, asesoría y documentación para prevenir o denunciar los abusos cometidos contra sus derechos humanos. La mayor parte de los usuarios de estas instalaciones, ya vienen de haber intentado cruzar la línea fronteriza, o se quedaron sin dinero; a veces por las tardes sus familiares que se encuentran en Estados Unidos de América llaman con la intención de obtener información sobre ellos.

Para promocionar los servicios prestados, la iglesia entrega volantes una o dos veces por semana, difunde información en las misas, y en el interior del templo mantiene de forma permanente afiches informativos. Del 22 de enero del 2010 al 23 de febrero del 2011 había atendido a 69 niños y niñas migrantes, el 88.4\% de la niñez atendida era originaria del mismo México, el $8.7 \%$ de Guatemala y el 2.9\% de Honduras.

\section{ACCIONES Y ESPACIOS QUE VULNERAN A LA NIÑEZ MIGRANTE}

La niñez migrante cuando llega al área urbana de Altar, se encuentran en un espacio, donde su contexto social es producto de las «formas de significación institucionalizadas que adopta [esta]

5 El DIF Municipal es la única instancia gubernamental que tiene dentro de sus funciones principales, brindar apoyo a la niñez migrante, entre las iniciativas implementadas en Altar se encuentran el otorgarles un pasaje diario a los que solicitan retornar a sus lugares de origen. Cuando requieren alimento o un espacio donde dormir son transferidos al CCAMYN, y la atención médica, es prestada en coordinación con el centro de salud o médicos particulares. 
sociedad en el pensar, en el decir, en el hacer, en el juzgar» (Baeza R 2011: 33) sobre la presencia de migrantes que van de paso. Así como el resultado de las relaciones sociales que han establecido, entre transeúntes y vecinos.

Su llegada podría pasar desapercibida, pero el objetivo que tienen los convierte en una mercancía valiosa para los enganchadores ${ }^{6}$ (que se dedican a ofrecer el servicio de guía), quienes los esperan a pocos metros de la plaza. Al bajar del autobús se les abalanzan, expresando frases como: ¿tú eres el recomendado?, ¿vas para el otro lado?, ¿tienes quién te lleve?, "vente conmigo que te voy a dar buen precio», y hasta les hablan fuerte, los toman de la mano o les dicen, "veras como te vas a bañar y a dormir bien» (Cabrera, notas de campo, 2011).

En este alboroto, pueden ser sorprendidos, terminando donde menos lo esperan, solicitando dinero a sus familiares para ser dejados en libertad, trasladando droga (burreando), o siendo vendidos a las redes que se encargan de llevarlos a Estados Unidos de América, si tienen a alguien que pague los costos del viaje en aquel país.

Hay quienes ya vienen prevenidos sobre la forma en que los van a recibir, Isidro fue uno de ellos, con 16 años de edad viajó solo desde el Estado de Guerrero, al ser entrevistado explicaba que su padre le dijo: "cuando vayas no le creas a la gente que te llama, tú vas con quien vas y ya». Orientaciones como estas, contribuyen a que se formen una cierta idea del lugar donde estarán por un tiempo, antes de intentar cruzar la frontera, y que mantengan sus medidas de seguridad.

Así inician a entablar las primeras relaciones sociales, de forma un tanto caótica, pero las mismas se pueden ir transformando en positivas o negativas, y generando «un mínimo de reciprocidad, que puede ser amor, amistad, conflicto, enemistad [o] piedad» (Weber, 1984: 21). Cada quien tiene una experiencia única, hay quienes consideran buenas personas a los vecinos de Altar con los que se relacionaron, mientras otros como David, que fue detenido al llegar al pueblo, por un grupo de hombres que andaban sin uniforme, ni

6 En los lugares de paso, ubicados en la línea fronteriza entre México y Estados Unidos de América, se les denomina «enganchadores» a quienes se dedican a ofrecer el servicio de guía para ayudarles a cruzar la frontera, a las personas que viajan por su propia cuenta (sin pagarle a las organizaciones que trafican con los migrantes). Por lo general estos enganchadores son parte de la estructura mafiosa, y lo que pretenden es aprovecharse de sus clientes. 
pistola, pero en una patrulla policial, y le quitaron 2.000 pesos, las perciben muy violentas y corruptas.

No se puede negar el avance que ha existido en la protección de los migrantes, lo que ha sido posible gracias a las acciones de las instituciones gubernamentales que se encargan de cumplir esa función, al papel protector que ha jugado la iglesia católica mexicana, a las iniciativas de la sociedad civil organizada, y las actividades individuales de ciertos ciudadanos. Pero esto no ha logrado eliminar los prejuicios que se tienen contra este sector poblacional en movimiento, que constituye la otredad, y es visto como peligroso (Velázquez García, 2008: 43), tratado despectivamente de Oaxaca, Oaxaquita, sucio, transmisor de enfermedades, alcohólico, irrespetuoso y destructor de los bienes comunales.

Cristina $^{7}$ que es voluntaria del CCAMYN, tiene bien claro lo que no le gusta de ellos, al ser entrevistada lo expresaba de la siguiente manera:

No importa que usen la plaza, lo que no estoy de acuerdo es que son muy sucios, si están sentados aquí, ellos tiran el sucio allí, aunque esté el bote de la basura a la par. También son tomadores, y no respetan, porque yo voy por la calle y les digo con permiso pero no se quitan, entonces les grito «voy a pasar» y se quitan; me molesta que no cuiden, destruyen lo poquito que nosotros tenemos, me molesté cuando pusieron rejas en la iglesia porque no es justo que lo hagan por ellos.

\section{LA DISPONIBILIDAD DE ALIMENTOS}

En el área urbana de Altar, los sitios que la niñez migrante prefiere para satisfacer sus necesidades alimenticias, son las pequeñas tiendas improvisadas que se encuentran a orilla de la carretera internacional, o cercanas a los lugares donde duermen, y algunas casas de huéspedes, en especial las que incluyen el alimento dentro de su paquete de servicios ofrecidos.

7 Los nombres de la niñez y las personas adultas que no desempeñaban un cargo de autoridad al momento de ser entrevistadas, han sido modificados para proteger su identidad. 


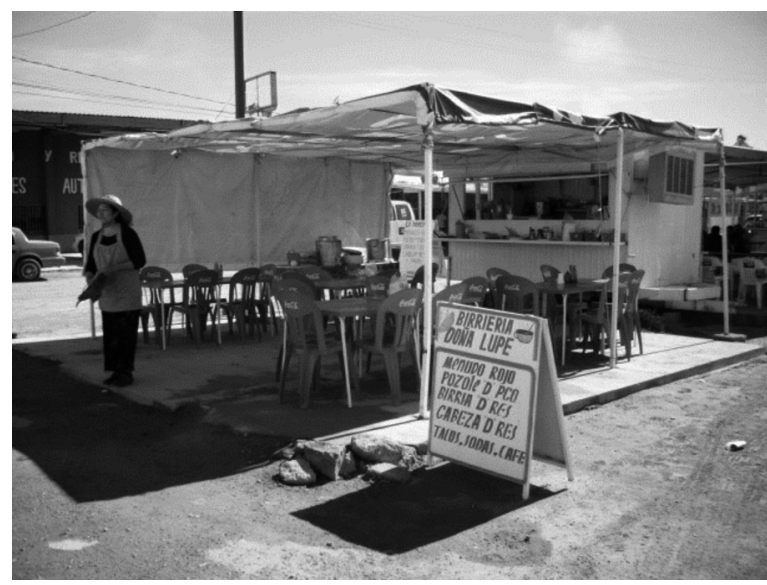

Fotografía: Mario Alexander Cabrera Duarte.

Una de las desventajas que tienen los consumidores de los locales improvisados ubicados a orilla de la carretera internacional, es la exposición constante al polvo que sufren los alimentos, lo que puede afectar su salud.

La disponibilidad de los recursos financieros necesarios (Beck, 1998: 41) les evita el riesgo (a quienes los poseen) de quedarse sin alimento en la localidad, permitiéndoles acceder a la cantidad y al tipo de producto que desean consumir, seleccionando los lugares que consideran más adecuados para sus gustos e higiene. En cambio, los que carecen de ellos, buscan sus propias estrategias que les lleven a mitigar el hambre, entre las que se encuentran cocinar donde están hospedados, comer solo una vez al día, preferir los productos como las galletas, bebidas gaseosas o bocadillos embolsados, pedirle comida a los habitantes del lugar o asistir al CCAMYN.

Al comprar los productos alimenticios para prepararlos donde están hospedados, se enfrentan a dos situaciones que no son muy agradables para ellos, la primera tiene que ver con su alto costo en comparación al valor que tienen en los lugares de donde vienen, por ejemplo, mientras en Altar un kilo de tortilla vale 20 pesos, Amalia una niña migrante originaria de Juquila, Oaxaca lo compraba en su pueblo a 9.50; la segunda situación se refiere al lugar donde preparan los alimentos, esta práctica fue observada en una de las casas de huéspedes visitadas, que tenía la cocina junto a las literas y se encontraba separada por madera contrachapada vieja, contaba con una estufa pequeña, la que había sido colocada sobre la única 
mesa que compartía el espacio con dos recipientes en los que se almacenaba el gas para cocinar, el entorno era insalubre, carecía de agua y estaba poco iluminado.

Angelina, quien es propietaria de la Farmacia Altarcito, al ser entrevistada expresó que la alimentación es uno de los factores que más incide en la salud del migrante. Según ella, hace falta un mayor control sanitario a los puestos de comida, nadie sabe la forma en que preparan los alimentos los negocios móviles que venden en la plaza y sus alrededores, eso expone a los consumidores ante enfermedades generadas por una manipulación incorrecta de los mismos.

Los otros negocios (hoteles y restaurantes) que tienen una mejor infraestructura, tampoco garantizan totalmente que sus productos no van afectar la salud de sus clientes, debido a que en ellos de acuerdo a la Secretaría de Salud Pública (2011: 1) puede existir fauna nociva en la cocina, carecer de buenas prácticas de salud e higiene, conservar los alimentos perecederos bajo temperaturas inadecuadas y utilizar los que ya han caducado, o no realizar análisis microbiológicos a los productos terminados.

La situación se agrava con la escasez de agua, lo que limita la existencia de sitios que estén disponibles para lavarse las manos antes y después de comer, aumentando la exposición de la niñez migrante a enfermedades que pueden evitarse, como las infecciones estomacales o la intoxicación por consumo de alimentos contaminados, siendo el pollo y la salsa unos de los principales generadores de estos problemas, en especial durante el verano, cuando se dañan muy rápido al carecer de la refrigeración adecuada.

\section{LAS CONDICIONES EN QUE DUERMEN}

Las casas de huéspedes junto a los hoteles se han convertido en los espacios más frecuentados por la niñez migrante para pasar la noche. Los servicios que brinda el CCAMYN gracias a la solidaridad de los ciudadanos, son utilizados en última instancia, cuando se ha terminado el dinero y el que migra no tiene a nadie que responda económicamente por él en Estados Unidos de América; es una forma de evitar quedarse durmiendo en la calle, a la intemperie, expuesto a la delincuencia común y a la mafia. 
De los 21 niños y niñas migrantes entrevistados, solo 1 eligió el lugar donde se quedaría a dormir, todos los demás dependían y obedecían a la decisión tomada por el coyote, o familiar que los llevó hasta Altar, y cuando viajaban totalmente solos, estaban limitados a las sugerencias que les daban los habitantes locales. Mostrando una obediencia "condicionada, por la voluntad de eludir las penas $\mathrm{u}$ otras consecuencias de [su] insumisión» (Simmel, 1989: 148), que se dan en el entorno al que llegaron, y de las que ya contaban con algún tipo de información previa.

Los hoteles cobran entre 350 y 450 pesos por pasar la noche en una habitación que cuenta con dos camas, televisor sin control remoto, ${ }^{8}$ ventilador o aire acondicionado, toallas, sanitario con papel higiénico y baño privado, en la que pueden dormir hasta cuatro personas. En cuanto a la higiene se consideran más seguros que las casas de huéspedes, pero en sus instalaciones los menores migrantes, están expuestos a situaciones que les pueden vulnerar, entre las que se encuentran la trata de personas y el hostigamiento sexual.

Amalia, expresó que ella se sentía incomoda en el hotel que se hospedaba, por las palabras que le decían los hombres con los que viajaba y compartía la habitación. Entre los comentarios que hizo se encontraba el siguiente: "hablan de sexo y yo no sé nada de eso, me dicen que si ya tuve relaciones, luego hablan de cosas que no puedo decir».

Referente a las casas de huéspedes, la forma en que han sido acondicionadas, es uno de los aspectos de gran utilidad para intentar conocer la vulnerabilidad social de la niñez migrante. En ellas se expresa la interacción de las culturas desiguales (Giménez, 2008: 24) a través de las relaciones sociales establecidas, entre el que presta el servicio siendo miembro del entorno que habita, y quien lo recibe en condición del otro.

Algunas representan verdaderos microsistemas, mientras la mayoría son casas con habitaciones comunes que han sido dedicadas a prestar el servicio de hospedaje. Las primeras son una minoría, están bien organizadas, sus instalaciones incluyen servicios de alimentación y hospedaje; además de santuarios dedicados a la Virgen de Guadalupe, abarrotería, venta de medicamentos para

8 Cuando el huésped desea utilizar el control remoto del televisor, debe dejar un depósito de 100 pesos en la recepción del hotel. 
tratar las dolencias más comunes, y equipo de uso personal que es de utilidad al desplazarse por el desierto.

En cambio, las que conforman la mayoría se caracterizan por sus condiciones precarias, en una habitación pueden alojar en total hacinamiento a 20 personas durante la temporada alta, las que deben compartir con los huéspedes de otras habitaciones las duchas y sanitarios. Aquí la niñez migrante no acompañada se expone a la perdida de sus pocas pertenencias, y a las molestias causadas por los migrantes adultos, entre los que se encuentran, aquellos que por la noche bajo los efectos de las drogas y el alcohol se ponen a jugar cartas, hacer ruido, y agredirles de forma verbal o física.

En estos espacios las drogas son un grave problema, los prestadores del servicio de hospedaje se quejan, porque les roban las bombillas del alumbrado eléctrico para quemar el crack, el que es vendido por los que se encargan del narcomenudeo, con el argumento de que les hará aguantar más la travesía en el desierto.

\section{EL ACCESO A LOS SERVICIOS DE SALUD}

La atención medica que puede recibir el migrante durante su recorrido, es de vital importancia para proteger su salud, que corre el riesgo de verse afectada por problemas sufridos en los lugares de paso, o al intentar cruzar la frontera. Cuando esto último sucede retornan al área urbana más cercana donde pernoctaron, en busca de ayuda para sanar sus padecimientos, o regresar a sus comunidades.

En el caso de la niñez migrante no acompañada que pernoctan en el área urbana de Altar, entre las dolencias que presentan se encuentran las diarreas, vómitos y los dolores de cabeza por trastornos estomacales, a las niñas se les adelanta la menstruación debido a la tensión nerviosa, y el cambio de temperatura les genera problemas respiratorios o deshidratación.

Su situación es más difícil cuando retornan de haber intentado cruzar la frontera; la exposición a temperaturas extremas (superior a los 45 grados centígrados en el día o bajo 0 por la noche), el dormir a la intemperie, la escasez de agua y alimentos o el consumo de los que han sido preparados de forma inadecuada, el uso de ropa sucia por varios días, las agresiones físicas, el caminar grandes distancias, y correr en medio del desierto cuando la Patrulla 
Fronteriza los persigue, contribuye a que presenten «ámpulas en los pies, deshidratación, fatiga muscular, parásitos, insolación» (Valdéz Gardea, notas de campo 2006), dolor estomacal, gastritis, diarreas, micosis, infecciones en las vías urinarias, dolor en la rodilla, inflamación en las piernas y alergias en la piel ${ }^{9}$.

Al necesitar atención médica, pueden acudir a los cuatro médicos privados y para comprar medicamento a las 8 farmacias que existen en la localidad, o recibir los servicios de forma gratuita a través del Centro de Salud Municipal, la Clínica Móvil de la Cruz Roja Mexicana, el DIF municipal, el CCAMYN, y en ocasiones mediante las jornadas médicas para migrantes implementadas por la DGAMI.

El Centro de Salud Municipal lo utilizan cuando tienen una emergencia, porque si el caso no es grave, para poderles atender, les exigen hacer una cita previa. Al necesitar medicamentos les brindan las recetas, y en los archivos de la institución no dejan ningún tipo de registro sobre la persona atendida.

La Clínica Móvil de la Cruz Roja Mexicana, es la más accesible a la niñez migrante no acompañada, sus servicios humanitarios los ha prestado durante varios años pero de forma temporal, aunque las instalaciones se cierran a las 8 de la noche, brinda atención prehospitalaria las 24 horas del día. Se encuentra equipada para realizar el chequeo de los signos vitales, el nivel de azúcar en la sangre, la curación de heridas y la entrega de medicamento para tratar problemas estomacales, contusiones, dolores y la deshidratación.

La jornada médica, es una iniciativa del Estado de Sonora dirigida a los migrantes, se implementa en el área urbana de Altar una vez al año de 9 de la mañana a 2 de la tarde; en ese tiempo la DGAMI mueve todo un equipo de profesionales, entre los que se encuentran médicos generales, oftalmólogos, microbiólogos, odontólogos y sus asistentes.

Ellos le brindan información a los migrantes sobre los diferentes tipos de enfermedades que pueden sufrir durante el recorrido, y les ofrecen la oportunidad de practicarse gratuitamente exámenes para la detección del cáncer, diabetes, VIH, y presión alta. Sus servicios prestados también incluyen atención odontológica, la vacunación

9 La información fue obtenida de los datos proporcionados por la Cruz Roja Mexicana delegación Sonora, el Programa Camino a Casa del SEDIF Sonora con sede en Nogales, y las entrevistas realizadas durante el trabajo de campo, los que se contrastaron con los ofrecidos por el Seminario Niñez Migrante de El Colegio de Sonora. 
universal, medicamentos, preservativos, y consulta externa; cuando se llega a detectar algún problema grave, el paciente es remitido a las dependencias de la Secretaría de Salud, donde es atendido y se le hacen todas las sugerencias correspondientes de acuerdo a la enfermedad que padece.

El DIF municipal y el CCAMYN también desarrollan acciones orientadas a proteger la niñez migrante no acompañada, ambas instancias les facilitan el acceso a los servicios de salud o la obtención de medicamentos. Sin embargo, la situación en que viajan, el estar bajo el poder de los guías o coyotes, el no contar con los fondos necesarios, el temor a ser detenidos por migración, o exponerse mucho más a la delincuencia común y la mafia, hace que utilicen con mayor frecuencia la automedicación para tratar de aliviar sus padecimientos; en estos casos, las farmacias son indispensables porque proveen el producto que necesitan, y les orientan sobre el medicamento que pueden ingerir.

Entre los productos que consumen se encuentran los analgésicos, antigripales, sales rehidratantes, ungüentos, relajantes musculares, antimicóticos, talcos y vendas. Los antibióticos eran lo que más usaban con la intención de contrarrestar las infecciones estomacales, tos, resfriados, y evitar las infecciones al sufrir una herida, pero a partir del 2010 con la nueva ley de salubridad se restringió su venta solo a los que portaran receta médica.

\section{CONSIDERACIONES FINALES}

La vulnerabilidad social como producto de la acción humana, en los espacios donde llega a pernoctar de forma temporal la niñez migrante no acompañado, se construye, transforma y mantiene, debido a la combinación de diversos aspectos, que influyen indirecta o directamente en el encuentro y las relaciones sociales creadas entre ellos y los vecinos de la localidad.

En el área urbana de Altar, los aspectos que inciden de manera indirecta son: las condiciones en que está migrando la niñez, las acciones antiinmigrantes de Estados Unidos de América que han sellado las zonas tradicionales de cruce, la limitada aplicación de medidas preventivas para protegerles, la imagen estereotipada que se ha difundido del migrante indocumentado, y la invisibilidad de 
la que son objeto por parte de las autoridades y la ciudadanía en las zonas fronterizas.

Los aspectos que influyen directamente en su vulnerabilidad, generados en los tres tipos de servicios descritos, se pueden resumir de la siguiente manera: en el servicio de alimentación, son el alto costo de cada porción de alimento, el consumo de alimentos mal preparados o contaminados, la falta de buenas prácticas de salud e higiene, junto a la carencia de estudios clínicos al personal que los prepara, la existencia de producto caduco, o su conservación a temperatura deficiente (Secretaría de Salud Pública, 2011).

La indefensión en que se puede encontrar este sector migratorio, al hacer uso del servicio de hospedaje, se vincula a la dependencia que tienen de otras personas para seleccionar el lugar donde van a dormir, el hacinamiento en que pasan la noche durante la temporada alta, la inseguridad generada en los lugares donde se quedan, debido a la ausencia de medidas preventivas contra desastres naturales o agresiones entre personas que ponen en riesgo la vida.

Referente a la salud, la misma se ve afectada por aspectos negativos vinculados a la alimentación y los espacios donde duermen, pero además de eso por la exposición al consumo de drogas. Cuando sufren algún tipo de dolencia, la situación se agrava debido a la inexistencia de un programa permanente de atención médica, que tome en cuenta sus necesidades y las condiciones en que viajan.

\section{BIBLIOGRAFÍA}

Almeida Acosta, E. (2009): «Comunidad e identidad: Procesos psicosociales para entender la vulnerabilidad social». METAPOLÍTICA, Noviembrediciembre (67), pp. 65-68. Obtenido de http://web.ebscohost.com/ehost/ pdfviewer/pdfviewer?vi $\mathrm{d}=4 \&$ hid=113\&sid=f3573e50-d497-474a-aa9ebc907e5b5cad\%40sessionmgr 111.

Alto Comisionado de las Naciones Unidas para los Refugiados. (2009): La protección internacional de las niñas y niños no acompañados o separados en la frontera sur de México. México D.F, México: Oficina regional de ACNUR para México, Cuba y América Central. Obtenido de http://www. acnur.org/biblioteca /pdf /6684.pdf.

AmNistía internacional. (2010): Victimas invisibles: Migrantes en movimiento en México. Madrid, Editorial Amnistía Internacional. 
Ayuntamiento de Altar. (2010): Negocios del área urbana de Altar. Altar, Sonora.

BaEza R, M. A. (2011): Elementos básicos de una teoría fenomenológica de los imaginarios sociales. En F. Randazzo, J. L. Pinto, J. R. Coca, \& J. A. Valero Matas, Nuevas posibilidades de los imaginarios sociales (págs. 3142). España: Asociación Cultural Tremn. Obtenido de http://www.tremn. org/documents/ Nuevas\%20posibilidades\%20def.pdf.

BEсK, U. (1998): La sociedad del riesgo: Hacia una nueva modernidad. Barcelona, España, A\&M Gráfic, S.L.

Busso, G. (2001): Vulnerabilidad social: Nociones e implicancias de políticas para Latinoamérica a inicios del siglo XXI. Ponencia presentada en El seminario internacional "Las diferentes expresiones de la Vulnerabilidad Social en América Latina y el Caribe», 20 y 21 de Junio. Santiago de Chile. Obtenido de http://www.redadultosmayores.com.ar/buscador/files/ORGIN011.pdf.

Bustamante, J. A. (2004): Nuevas tendencias y desafíos de la migración internacional. En J. Santibáñez Romellón, C. Fonte Ávalos, \& M. Á. Castillo García, Memorias del seminario permanente sobre migración internacional (págs. 293-331). Tijuana, Baja California, El Colegio de la Frontera Norte.

CAREAGA, K. (2009): Construcción de la vulnerabilidad y agencia de las mujeres ante las enfermedades de transmisión sexual (ETS) en el proceso migratorio de Altar, Sonora, 2006-2007. Tesis de Doctoraro en Ciencias Sociales. Hermosillo, Sonora, México, El Colegio de Sonora.

Chavez, L., \& Menjivar, C. (2010): «Children without borders: A Mapping of the Literature on Unaccompanied Migrant Children to the United States». Migraciones internacionales, 2 (3), pp. 71-111. Obtenido de http:// www2.colef. mx/migraciónesinternacionales/revistas/MI18/n18-071-111. pdf.

Comisión Económica para América Latina y el CARibe. (2001): Informe de la reunión de expertos. Seminario internacional sobre las diferentes expresiones de la vulnerabilidad social en América Latina y el Caribe, 20 y 21 de junio. Santiago de Chile.

De Sauza Minayo, M. C. (1995): Etapas del trabajo de campo. En Ídem, El desafío del conocimiento: investigación cualitativa en salud (págs. 91-115). Buenos Aires, Argentina, Lugar Editorial S.A.

Denman, C. A., \& Haro, J. A. (2000): Introducción. En Ídem, Por los rincones. Antología de métodos cualitativos en la investigación social (págs. 9-55). Hermosillo, México, El Colegio de Sonora.

Denzin, N. K. (2002): Un punto de vista interpretativo. En C. A. Denman, \& J. A. Haro, Por los rincones: Antología de métodos cualitativos en la investigación social (págs. 147-205). Hermosillo, México, El Colegio de Sonora.

FigueroA, J. G. (2003): "La evaluación ética de proyectos de investigación desde una perspectiva de género». Revista Mexicana de Bioética, 1 (1), pp. 65-76. 
Fondo de las Naciones Unidas PaRa la InFancia. (2006): Convención sobre los Derechos del niño. Madrid, España, Nuevo Siglo. Obtenido de http:// www.unicef.es/ derechos/docs/CDN_06.pdf.

Galindo, J. (1987): «Encuentro de subjetividades, objetividad descubierta. La entrevista como centro de trabajo etnográfico». Estudios sobre las Culturas Contemporáneas, 1 (3), pp. 151-183.

Gallo Campos, K. I. (2004): Niñez migrante en la frontera norte: Legislación y procesos. México D.F: DIF/UNICEF.

García Vidales, M. A., Molina Nava, M. d., \& López Vega, R. (2008): Menores migrantes en México. En G. C. Valdéz Gardea, Achicando Futuro (págs. 49-70). Hermosillo, Sonora, México, El Colegio de Sonora.

Giménez, G. (2008): «Cultura, identidad y memoria: Materiales para una sociología de los procesos culturales en las franjas fronterizas». Frontera Norte, 21 (41), pp. 7-32.

GonZÁlez FAGOAGA, J. E. (2009): «La vulnerabilidad de los grupos migrantes en México». Migraciones internacionales, 5 (3), pp. 233-239.

Instituto Nacional de Estadística y Geografía. (15 de Febrero de 2011): Prontuario de información geográfica municipal de los Estados Unidos Mexicanos: Altar, Sonora, clave geoestadística 26004. Obtenido de http://www. inegi.org.mx/ sistemas/mexicocifras/datos-datosgeograficos/

Instituto Nacional de Migración. (2010): Boletín mensual de estadísticas migratorias. México D.F., Centro de Estudios Migratorios. Obtenido de http://www.inm.gob. mx/estadísticas/2010/agosto/BoletinEst2010.pdf.

Instituto Nacional de Migración. (17 de Abril de 2011): Eventos de repatriación de mexicanos desde Estados Unidos, según entidad federativa de repatriación, grupos de edad y sexo, 1998-2010. Obtenido de http://www.inm. gob.mx/index. php/page/Series_Historicas

Kaztman, R. (2000): Notas sobre la vulnerabilidad social. $5^{\circ}$ Taller regional la medición de la pobreza: Métodos y aplicaciones, Del 6 al 8 de Junio. Aguascalientes. Obtenido de http://www.eclac.cl/deype/mecovi/docs/TALLER 5/24.pdf.

LizÁrraga García, B. (2000): Altar y los altareños. Altar, Sonora, España, Ayuntamiento de Altar.

López CAstro, G. (2005): Niños, socialización y migración a Estados Unidos en Michoacán. Ponencia Conferencia Internacional Perspectivas mexicanas y estadounidenses en el estudio de la migración internacional. Obtenido de http://cmd.princeton.edu/papers/wp0502d.pdf.

Martín-Crespo Blanco, M. C., \& Salamanca Castro, A. B. (2007): «El muestreo en la investigación cualitativa». Nure Investigación, Marzo-Abril (27), pp. 1-4.

Massey, D. S., Arango, J., Hugo, G., Kouaduci, A. Pellegrino, A., \& Taylor, J. E. (2008): «Teorías de migración internacional: una revisión y aproximación». REDCE, 10 , pp. 435-478. 
Mendoza Rockwell, E. N. (2006): La intimidad del desierto: Moral, identidad y tráfico de drogas en un lugar complicado. Tesis de licenciatura en relaciones internacionales.

Pizarro, R. (2001): La vulnerabilidad social y sus desafíos: una mirada desde América Latina. Santiago de Chile, Chile, CEPAL/ECLAC. Obtenido de http://www. eclac.org/publicaciones/xml/3/6553/lcl1490e.pdf.

RANGel Gómez, G. (2008): Niñez migrante no acompañada en la frontera norte: retos y desafíos. En G. C. Valdéz Gardea, Achicando futuros: Actores y lugares de la migración. Hermosillo, Sonora, México, Colegio de Sonora.

Ruíz, O. (2001): «Riesgo, migración y espacio fronterizo: Una reflexión». Estudios demográficos urbanos, pp. 257-284.

Santos Villareal, G. M., \& Ávila Loya, P. (2009): La migración infantil: un problema acuciante. México D.F., Centro de documentación, información y análisis. Obtenido de http://www.diputados.gob.mx/cedia/sia/spe/ SPE-ISS-21-09.pdf.

Secretaría de Salud Pública. (2011): Oficio número.: SSP/DGPRS/2010-1301. Dirección General de Protección Contra Riesgos Sanitarios, Hermosillo.

Simmel, G. (1989): Sociología 1: Estudios sobre las formas de socialización. España: Alianza Editorial.

SufCliffe, B. (1998): Nacido en otra parte. Bilbao, Marra publicidad.

TAYlor, S., \& Bogdan, R. (1992): El trabajo de los datos: Análisis de los datos en la investigación cualitativa. En Ídem, Introducción a los métodos cualitativos de investigación (págs. 152-176). Barcelona, España, Paidós.

TRuax, E. (2006): Altar, la nueva ruta de los migrantes: El poblado, ubicado en el desierto de Sonora, ofrece todos los servicios para el cruce. Obtenido de http:// colombiareport.ss.uci.edu/webdocs/lanuevarutadelosinmigrantes. pdf.

Tuirán, R., \& Luis, Á. J. (2010): La migración México-Estados Unidos, 19402010. En F. M. Ángel Castillo, \& G. Verduzco Alba, Migraciones internacionales (págs. 93-133). México. D.F., México, El Colegio de México.

Valdéz Gardea, G. C. (Mayo de 22 de 2007): Altar, Sonora: Geografías rurales olvidadas. Obtenido de Seminario niñez migrante: http://seminarionm. blogspot. com/search/label/ART\%C3\%8DCULOS\%20P

VAldÉz Gardea, G. C. (2008): Presentación. En Ídem, Achicando futuro: actores y lugares de la migración (págs. 15-21). Hermosillo, Sonora, México, El Colegio de Sonora.

VAldéz Gardea, G. C. (22 de Mayo de 2009): Negligencia al contar. Obtenido de Seminario Niñez Migrante: http://seminarionm.blogspot.com/search/ label/ART \%C3\%8DCULOS\%20PERIOD\%C3\%8DSTICOS

Velasco, H., \& Díaz de la RADA, Á. (2006): La lógica en la investigación etnográfica: Un modelo de trabajo para etnógrafos de escuela. Madrid, España, Editorial Trotta, S.A.

Velázouez García, M. A. (2008): «La construcción de la imagen de México en Estados Unidos desde una perspectiva de riesgo». Frontera Norte, 20 (39), pp. 37-68. 
Von der Borch, M. (2011): Altar, Sonora (2000-2006): Elementos para la construcción de una mirada desde el interior. Hermosillo, Sonora, México, Material sin públicar.

WeBer, M. (1984): La acción social: Ensayos metodológicos. Barcelona, España, Nova-Gráfik.

Yánez Moreno, P., \& Hernández Martínez, E. O. (2010): De oruga a mariposa: La constitución de los ritos de paso en jóvenes migrantes. Ponencia presentada en el Coloquio binacional sobre Desarrollo Humano Transfronterizo en la Región Sonora-Arizona realizada el 27 y 28 de mayo. Nogales, Sonora.

\section{Entrevistas y notas de campo}

Amalia [entrevista], 2011, por Mario Alexander Cabrera [trabajo de campo], «La vulnerabilidad social de los menores migrantes no acompañados, en el área urbana del municipio de Altar, Sonora, México durante los años 2010-2011», Programa Camino a Casa de Nogales.

Angelina [entrevista], 2011, por Mario Alexander Cabrera [trabajo de campo], «La vulnerabilidad social de los menores migrantes no acompañados, en el área urbana del municipio de Altar, Sonora, México durante los años 2010-2011», Programa Camino a Casa de Nogales.

CABrera [Notas de campo], 2011, por Mario Alexander Cabrera [trabajo de campo], «La vulnerabilidad social de los menores migrantes no acompañados, en el área urbana del municipio de Altar, Sonora, México durante los años 2010-2011», Altar.

CRISTINA [entrevista], 2011, por Mario Alexander Cabrera [trabajo de campo], «La vulnerabilidad social de los menores migrantes no acompañados, en el área urbana del municipio de Altar, Sonora, México durante los años 2010-2011», Altar.

DAvid [entrevista], 2011, por Mario Alexander Cabrera [trabajo de campo], «La vulnerabilidad social de los menores migrantes no acompañados, en el área urbana del municipio de Altar, Sonora, México durante los años 2010-2011», Programa Camino a Casa de Nogales.

Francisco [entrevista], 2006, por Gloria Ciria Valdéz Gardea [trabajo de campo], «Diagnostico del tráfico de menores y mujeres en el Estado de Sonora: Caso de estudio en los municipio de Agua Prieta, San Luis Río Colorado y Nogales. Recomendaciones de políticas públicas», Altar.

IsIDRo [entrevista], 2011, por Mario Alexander Cabrera [trabajo de campo], «La vulnerabilidad social de los menores migrantes no acompañados, en el área urbana del municipio de Altar, Sonora, México durante los años 2010-2011», Altar.

VALDÉz GARDEa [Notas de campo], 2006, por Gloria Ciria Valdéz Gardea [trabajo de campo], «Diagnostico del tráfico de menores y mujeres en el Estado de Sonora: Caso de estudio en los municipio de Agua Prieta, San Luis Río Colorado y Nogales. Recomendaciones de políticas públicas», Altar. 
